\title{
Lennox gastaut syndrome- A case report and review of treatment protocol
}

\author{
Poonam Prakash $^{1 *}$, SK Bhandari², Manab Kosala ${ }^{3}$ \\ ${ }^{1,3}$ Associate Professor, ${ }^{2}$ Professor and HOD, Dept. of Dental Surgery \& Oral Health Sciences, Armed Forces Medical \\ College, Pune, Maharashtra, India
}

*Corresponding Author: Poonam Prakash

Email: pnmprakash@yahoo.co.in

\begin{abstract}
Lennox-Gastaut syndrome (LGS) is a severe pediatric epilepsy syndrome characterized by mixed seizures, cognitive decline, and generalized slow $(<3 \mathrm{~Hz})$ spike wave discharges on electroencephalography. Seizures are often resistant to therapy. Atonic seizures result in dangerous drop attacks with risks of injury and impairment of the quality of life. The seizures are frequently resistant to multiple antiepileptic (AED) drugs. The non pharmacological treatments such as the ketogenic diet, vagus nerve stimulation, and epilepsy surgery should be considered when multidrug therapy is of limited help.

This paper aims to present a case report along with an updated comprehensive outline of LGS and the available medical, surgical and dental treatments. Further long term randomized controlled trials are required to compare efficacy of different therapeutic interventions.
\end{abstract}

Keywords: Lennox Gataut syndrome, Pediatric epilepsy, Ketogenic diet.

\section{Introduction}

Lennox Gastaut Syndrome is a pediatric epilepsy syndrome characterized by multiple types of seizures, mental retardation or regression, abnormal EEG with generalized slow spike and wave discharges. Seizures are often resistant to therapy. The management options include pharmacological and non pharmacological strategies like ketogenic diet, Vagus nerve stimulation and Focal Cortical Resection.

\section{Case Report}

A 20yr old girl reported to Central OPD at dept. of dental surgery with her parents for routine dental check up [Fig. 1, 2]. She was born of a non consanguineous marriage at Pune by a normal delivery. At $2 \frac{1}{2}$ months of age, she had an episode of seizures. At $4 \frac{1}{2}$ months of age, Valproic acid, Phenytoin \& Lorazepam were started. She showed delayed milestones. MRI of brain was done at 6 months of age. It revealed frontal lobe agenesis \& organic damage to the brain. Complete cardiothoracic check up was done which revealed mitral regurgitation.

She was diagnosed as a case of Lennox Gastaut Syndrome. She has been on antiepileptic medications since then. She is presently on Valproic acid and Topiramate. During the last 20 years sedatives were added \& removed from the regimen as per the requirements. She has irregular sleep pattern. Hysterectomy was done 06 months back. There is no history of similar problem in any of her family members.
Maxillofacial examination revealed anterior open bite \& mouth opening of $40 \mathrm{~mm}$. She keeps her mouth open all the time \& there is continuous drooling of saliva [Fig. 3]. She had divergent eyes \& broad bridge of nose with protruded maxillofacial complex.

Examination of the oral cavity revealed poor oral hygiene with multiple carious teeth. There was high arched palate \& malocclusion. Generalized gingival overgrowth was present [Fig 4].

Hematological investigations revealed $\mathrm{Hb}$ of 13 gm\%. Serum electrolyte was $148 \mathrm{mEq}$. ECG was confirmative of mitral regurgitation.

On basis of the history, clinical, dental \& investigative findings, a diagnosis of Lennox Gastaut Syndrome with Drug Induced Gingival Enlargement was made.

It was planned to carry out comprehensive dental treatment under general anaesthesia. Complete intraoral examination was carried out along with oral prophylaxis and extraction of all the carious teeth. Primary closure using 3-0 vicryl suture was done postextraction.

\section{Discussion \\ Clinical features}

Lennox Gastaut Syndrome is synonymous with childhood epileptic encephalopathy with diffuse slow spike waves, pediatric epilepsy syndrome, childhood epilepsy \& seizures with mental retardation.

It is a pediatric epilepsy syndrome constituting 1$4 \%$ of childhood epilepsies. It is characterized by 
mental retardation with multiple types of seizures. Tonic seizures or drop attacks in which the child suddenly falls on the ground \& injures himself. Atypical or absence seizures in which the child appears vacant or blank. During these episodes, the child drools, is unable to speak \& needs help for feeding. Tonic seizures are also seen in which there is stiffening of arms \& legs.

All children with this syndrome develop learning difficulties \& developmental delay. ${ }^{1,2}$

\section{Epidemiology \& Etiology}

LGS is rare, with an annual incidence of 0.2 2.8/10,000 births in European countries. LGS starts between 2 and 8 years (peak 3-5 years), slightly later in cryptogenic cases than in symptomatic ones. There is no racial difference. Males are affected more frequently than females.The mean age of epilepsy onset is 26-28 months.

Most children with LGS (75\%) have an underlying structural (symptomatic) brain abnormality. ${ }^{3}$ These include developmental cortical malformations, neurocutaneous syndromes, post hypoxic-ischemic insult, post meningitis/encephalitis, or metabolic encephalopathy.

The exact pathophysiology is not known. One hypothesis states that when anterior parts of the brain mature, there is excessive permeability in the excitatory interhemispheric pathways in the frontal areas [4]. Another study shows an association between LGS \& HLA (Human Lymphocyte Antigen) class I antigen B7.

The idiopathic form accounts for about 22-30\% of cases. The patient is free from neurologic \& neurophysiologic deficit prior to onset of symptoms. The problems appear during evolution of LGS generally after two years of age.

It is symptomatic in about $70-80 \%$ of the cases. It is associated with West Syndrome (infantile spasms) $\&$ seizures are more frequent. The causes are encephalitis, meningitis, brain malformations, birth injury \& frontal lobe agenesis.

It shows a mortality rate of $3-7 \%$. Death is often related to accidents. It shows high rate of injuries with atonic and tonic seizures. Patients should wear protective helmets with face guards.

Electroencephalogram is helpful when the child is not having obvious seizures. ${ }^{5}$ It shows bursts of irregular \& generalized spikes or waves followed by sinusoidal 35-400 millisecond slow wave which can be symmetric or asymmetric. The amplitude is high in anterior or frontal \& frontocentral areas.
MRI is the preferred investigation. CT scan is done if intracranial injury or hematoma is suspected due to head injury (tonic seizure).

Sometimes cause can be a genetic condition called Tuberous Sclerosis, meningitis or head injury. In about $50 \%$ of the cases cause can't be identified.

\section{Management}

The aim of the management protocol is to achieve complete seizure control; however, in many cases only reduction in seizure frequency and severity is possible. $^{6}$

The quality of life both for the patient and family can be improved by seizure control that will result in improved levels of alertness and reduced risks of injury. ${ }^{7}$

Treatment options include medical care and surgical intervention

\section{Pharmacological treatments}

The management is multidrug therapy, therefore it is of paramount importance to avoid drug related side effects and worsening of the associated comorbidities. This can be achieved with careful monitoring of drug levels, side effects and drug interactions 30 . The most common regimen used consists of such as Sodium Valproate, Lamotrigine, Topiramate, Phenytoin Sodium. ${ }^{8}$ Two monotherapy trials are recommended before polytherapy is considered. ${ }^{9,10}$

\section{Non-pharmacological treatments}

Despite the availability of many AEDs for the treatment of LGS, most patients fail to respond due prolonged duration of multiple drug usage. Other options include \& Ketogenic diet. ${ }^{11}$ Surgical options include Corpus callosotomy, ${ }^{12}$ Vagus Nerve Stimulation ${ }^{13}$ and Focal Cortical Resection.

\section{Ketogenic diet}

The ketogenic diet consists of a high fat low carbohydrate ratio. ${ }^{14}$ Up to $50 \%$ of patients achieve significant seizure reduction. ${ }^{15}$ Side effects include renal stones, hyperuricemia, acidosis, metabolic disturbance, vomiting, diarrhoea, and hyperlipidemia.

\section{Vagus nerve stimulation}

Vagal nerve stimulation has proven to have some effect in patients that are not candidates for epilepsy surgery. ${ }^{16}$ It improves the seizure control gradually with weekly increments of stimulation intensity. ${ }^{17}$ In addition, improvements in cognition and mood may be associated. ${ }^{18}$ The adverse effects include laryngeal irritation, drooling, hoarseness, dysphagia, dyspnea, 
and cough. ${ }^{19}$ No life-threatening side effects have been reported.

\section{Epilepsy surgery}

Surgical management includes partial or complete corpus callosotomy that results in significant reduction of the frequency of drop attacks. The improvements can be dramatic immediately after surgery with later recurrence. The side effects include decreased speech output, hemiparesis, gait disturbance, and disconnection syndrome. Partial corpus callosotomy (sectioning the anterior 2 thirds) involve better speech preservation; however, complete callosotomy is more effective. ${ }^{20}$ The procedure was also shown to reduce secondary generalized tonic-clonic seizures. ${ }^{21}$

\section{Special Dental Management}

The appointments should be kept in the morning \& and should be of short duration. Treatment of the individual should be carried out in his own chair. A special base can be used into which the wheelchair fits or a beanbag placed on the dental chair can be used to support the patient [Fig. 5]. A finger guard should be used to prevent any injury during the treatment phase if the patient develops an epileptic episode. Toothbrushes can be modified in the form of thicker handles so as to provide a better grip. Powered toothbrushes can also be used to perform the routine prophylaxis [Fig. 6]. It is very important to educate the guardians of the patient regarding importance of maintaining good oral hygiene.

Another option is treatment under general anaesthesia when the routine measures and modifications in treatment modalities do not achieve the desired results.

Prognosis is poor. Individual is dependant on others for lifetime. Some of them also develop chest infections which may shorten their life.

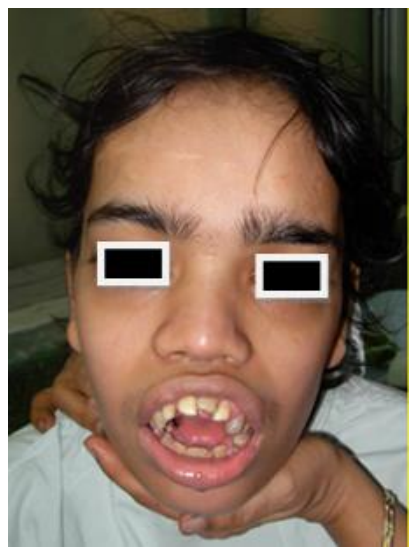

Fig. 1: Lennox Gastaut Syndrome (Frontal view)

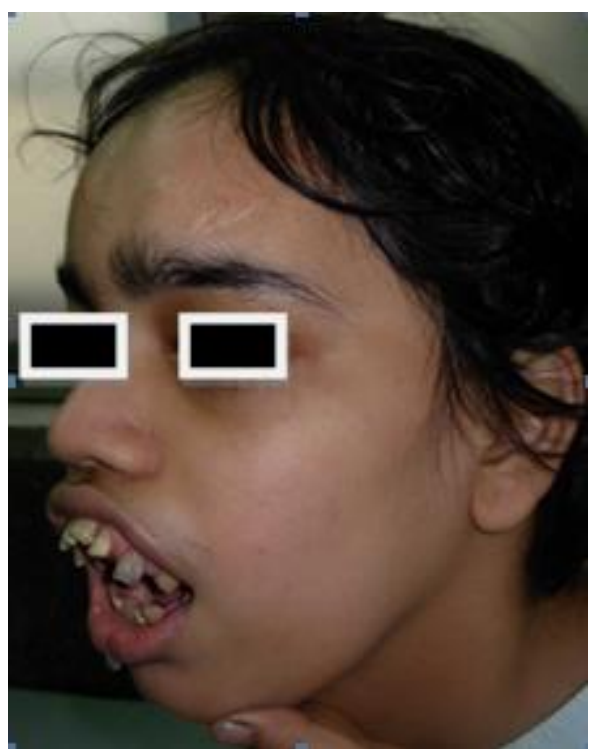

Fig. 2: Lennox Gastaut Syndrome (Profile view)

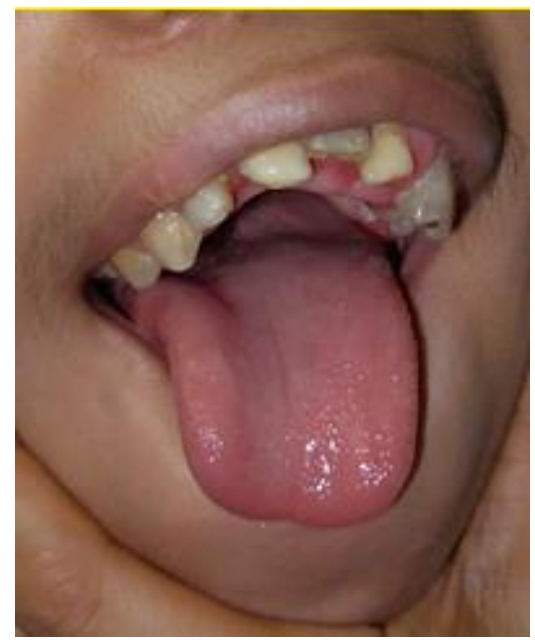

Fig. 3: Close up view showing open mouth \& drooling of saliva

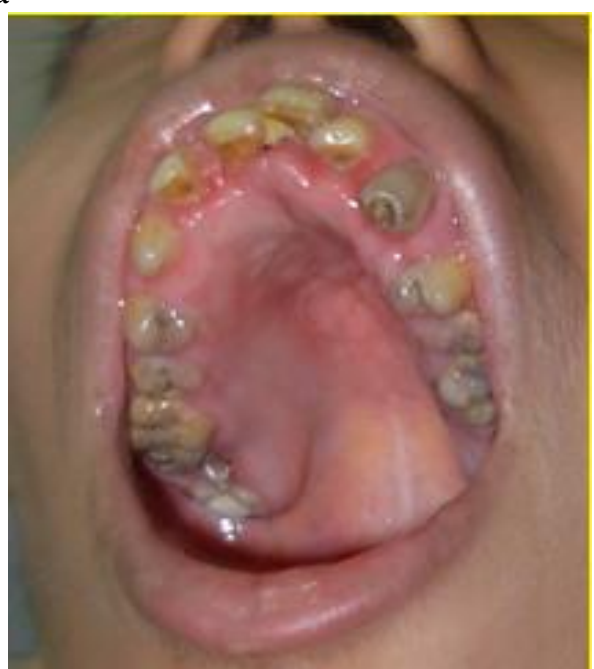

Fig. 4: Intraoral view showing gingival enlargement 


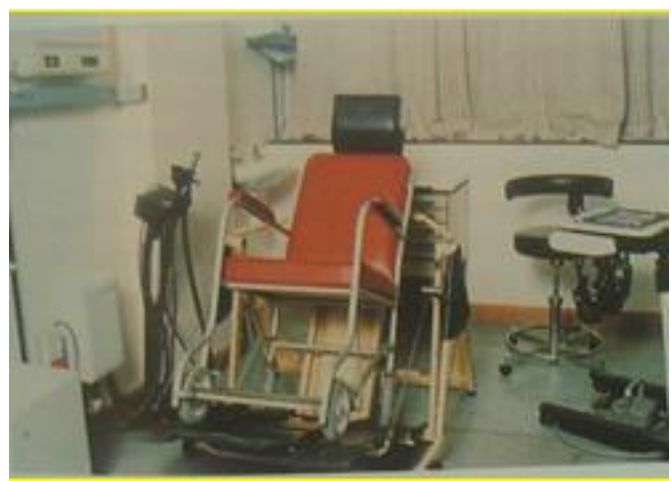

Fig. 5: Modified dental chair to fit wheelchair

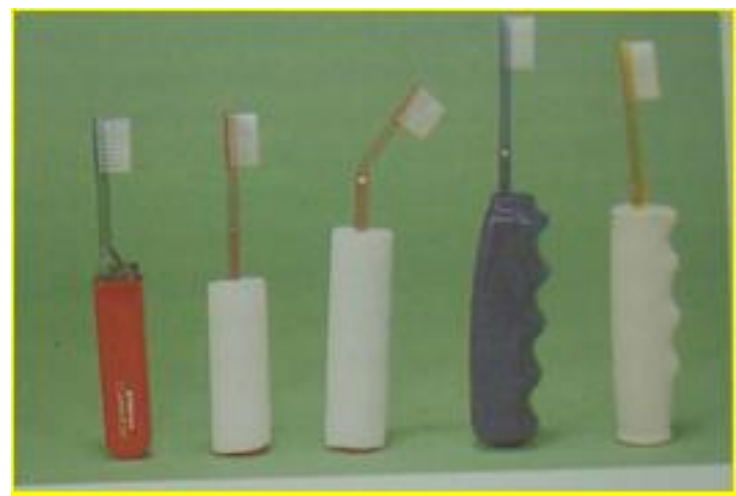

Fig. 6: Modified toothbrushes

\section{Prognosis}

Lennox-Gastaut syndrome generally has a poor prognosis. Most patients will have drug resistant epilepsy, and up to $80 \%$ of patients may continue to have daily seizures. ${ }^{22}$ In adult LGS, patients tonic seizures during sleep remain the major seizure type. $^{23}$ Evidence shows that the early use of newer AEDs could limit the cognitive dysfunction. ${ }^{24}$ In addition to multiple comorbidities, patients with LGS have an increased mortality rate of $5-17 \%$ mostly as a direct result of seizure related complications.

\section{The future}

\section{Neuroprotection}

Based on animal studies, some of the newer AEDs show possible neuroprotective activity in epilepsy. ${ }^{25}$ Although neuroprotection is successful only against some aspects of epilepsy, it might be a promising option in the treatment of refractory cases. However, to prevent epileptogenesis, interventions need to be directed against the processes implicated in the brain changes that underlie hyperexcitability and not only in the prevention of neuronal death. ${ }^{26}$

\section{Gene therapy}

Gene therapy is an innovative and promising alternative for the treatment of refractory epileptic patients, especially if inaccessible to surgery. ${ }^{27}$ Several gene targets could be used to correct the balance between inhibitory and excitatory aspects of epilepsy with recombinant viral vectors or transduction of neuropeptide genes. ${ }^{28}$ Although the proof of concept may have been established, further investigations are required to demonstrate a therapeutic role of gene therapy in epilepsy and to evaluate safety concerns and possible side-effects.

\section{Conclusion}

Dental treatment of the patients with various physical and mental disorders is a challenge for the dental surgeon. However the latest advancements in the field of dentistry along with the thorough understanding of the patient's status make it simple to provide the best possible treatment options to them by various modifications in the treatment procedures and equipment.

A better strategy is to explain regularly to the family that the aim of the treatment is to suppress the most severe seizures, to avoid additional comorbidities and to avoid heavy poly-therapy. The situation has to be regularly re-evaluated. As LGS is rare, case reports of atypical or unusual treatments may help other clinicians in deciding what type of treatment to use in difficult cases. The prognosis for complete seizure control is frequently poor. Further long term randomized controlled trials are required, particularly to compare different therapeutic interventions in terms of efficacy and tolerability.

\section{Source of funding \\ None.}

\section{Conflict of interest}

None.

\section{References}

1. Ferlazzo E, Nikanorova M, Italiano D, Bureau M, Dravet $\mathrm{C}$, Calarese T, et al. Lennox-Gastaut syndrome in adulthood: clinical and EEG features. Epilepsy Res 2010; 89: 271-7.

2. Arzimanoglou A, French J, blume WT, Cross JH, Ernst JP, Feucht M, et al. Lennox-Gastaut syndrome: A consensus approach on diagnosis, assessment, management, and trial methodology. Lancet Neurol 2009; 8: 82-93.

3. Ohtahara S, Ohtsuka Y, Yoshinaga H, Oka E, Yoshinaga $\mathrm{H}$, Sato M et al. Lennox-Gastaut syndrome: Etiological considerations. In: Niedermeyer E, Degen R, editors. The Lennox-Gastaut. 
4. Mima-T, Nagamine-T, Ikeda-A, Yazawa-S, Kimura-J, Shibasaki-H: Pathogenesis of cortical myoclonus studied by magnetoencephalopathy. Ann-Neurol 1998;43(5):598607.

5. Bare MA, Glauser TA, Strawsburg RH: Need for electroencephalogram video confirmation of atypical absence seizures in children with Lennox-Gastaut syndrome. J Child Neurol 1998;13(10):498-500.

6. Gallop K, Wild D, Verdian L, Kerr M, Jacoby A, Baker G, et al. Lennox-Gastaut syndrome (LGS): development of conceptual models of health-related quality of life (HRQL) for caregivers and children. Seizure 2010;19:23-30.

7. Carmant L, Whiting S. lennox-Gastaut syndrome: an update on treatment. Can J Neurol Sci 2012;39:702-11.

8. Alvarez N, Besag F, Iivanainen M: Use of antiepileptic drugs in the treatment of epilepsy in people with intellectual disability. J Intellect Disabil Res 1998;42(1):115.

9. Montouris GD. Rational approach to treatment options for Lennox-Gastaut syndrome. Epilepsia 2011;52:10-20.

10. Jan MM. Clinical review of pediatric epilepsy. Neurosci (Riyadh) 2005;10:255-64.

11. Caraballo-R, Tripoli-J, Escobal-L, Cersosimo-R, Tenembaum-S, Palacios- $C$ et al, Ketogenic diet: efficacy and tolerability in childhood intractable epilepsy. RevNeurol 1998;26(149):61-4.

12. Rougier-A, Claverie-B, Pedespan-JM, Marchal-C, LoiseauP: Callosotomy for intractable epilepsy. J Neurosurg Sci 1997;41(1):51-7.

13. Ben-Menachem E, Hellstrom K, Waldton C: Evaluation of refractory epilepsy treated with vagus nerve stimulation for up to 5 years. Neurol 1999;52(6):1265-7.

14. Bahassan NA, Jan MM. Ketogenic diet. Update and application. Neurosci (Riyadh) 2006;11:235-40.

15. You SJ, Kang HC, Ko TS, Kim HD, Yum MS, Hwang YS, et al. Comparison of corpus callosotomy and vagus nerve stimulation in children with Lennox-Gastaut syndrome. Brain Dev 2008; 30:195-9.

16. Karceski SC. Vagus nerve stimulation and Lennox-Gastaut syndrome: A review of the literature and data from the VNS Patient Registry. CNS Spectrums 2001; 6:766-70.

17. You SJ, Kang HC, Ko TS, Kim HD, Yum MS, Hwang YS, et al. Comparison of corpus callosotomy and vagus nerve stimulation in children with Lennox-Gastaut syndrome. Brain Dev 2008;30:195-9.

18. Grill MF, Ng YT. Dramatic first words spoken in 2 children after vagus nerve stimulation. Semin Pediatr Neurol 2010;17: 54-57.

19. Cyberonics Inc. Physician's manual, VNS Therapy Pulse Model 102 Generator. Houston (TX): Cyberonics Inc; 2002.

20. Maehara T, Shimizu H. Surgical outcome of corpus callosotomy in patients with drop attacks. Epilepsia 2001;42:67-71.

21. Nei M, O’Connor M, Liporace J, Sperling MR. Refractory generalized seizures: response to corpus callosotomy and vagal nerve stimulation. Epilepsia 2006;47:115-22.

22. Blume WT, David RB, Gomez MR. Generalized sharp and slow wave complexes. Associated clinical features and long-term follow-up. Brain 1973;96:289-306.

23. Ferlazzo E, Nikanorova M, Italiano D, Bureau M, Dravet $\mathrm{C}$, Calarese T, et al. Lennox-Gastaut syndrome in adulthood: clinical and EEG features. Epilepsy Res 2010; 89: 271-277.

24. Chieffo D, Battaglia D, Lettori D, Del Re M, Brogna C, Dravet C, et al. Neuropsychological development in children with Dravet syndrome. Epilepsy Res 2011; 95:8693.

25. Stêpieñ K, Tomaszewski M, Czuczwar SJ. Profi le of anticonvulsant activity and neuroprotective effects of novel and potential antiepileptic drugs - an update. Pharmacol Rep 2005;57:719-33.

26. Dichter MA, Cole AJ. Neuroprotection and antiepileptogenesis: where are we now? Neurol 2002;59(9S5):S36-S38.

27. Freese A, Kaplitt MG, O'Connor WM. Direct gene transfer into human epileptogenic hippocampal tissue with an adeno-associated virus vector: implications for a gene therapy approach to epilepsy. Epilepsia 1997;38:759-66.

28. Noe F, Nissinen J, Pitkänen A. Gene therapy in epilepsy: The focus on NPY. Peptides 2007;28:377-83.

How to cite: Prakash $\mathrm{P}$, Bhandari SK, Kosala M. Lennox gastaut syndrome- A case report and review of treatment protocol. IP Int $J$ Med Paediatr Oncol 2020;6(1):5-9. 